\title{
The Role of Urine Investigations in Urology Practice
}

\author{
Muhammad Ujudud Musa \\ Urology Unit, Department of Surgery, Federal Medical Centre Katsina, Katsina, Nigeria \\ Email: ujudud@gmail.com
}

Received 6 February 2015; accepted 9 April 2015; published 14 April 2015

Copyright (C) 2015 by author and Scientific Research Publishing Inc.

This work is licensed under the Creative Commons Attribution International License (CC BY).

http://creativecommons.org/licenses/by/4.0/

(c) (i) Open Access

\begin{abstract}
Urine examination is one of the important armamentarium in the practice of urology and it is a fundamental test that is usually carried out for urology patients, it can be as simple as dipstick analysis to a complex hormonal assays. Urine examination is done worldwide as an extension of physical examination which provides a lot of information about the pathology, and both physical and chemical analysis of urine are highly informative. There are several types of urine investigations, some of which include urinalysis, urine microscopy culture and sensitivity, urine microscopy for ova or cyst of parasites, urine cytology, urine tumour antigens assays, urine hormonal assays, urine toxicology, urine quantitative measurement and urine acid fast bacilli. Uses of urine examinations in urology could be diagnostic, such as renal function test, evaluation of heamaturia, stone diseases, urinary tract infections, urologic cancers and infertility or monitoring and for prognosis. Uses of urine investigations in practice of urology cannot be over emphasized as it has many revealing information of the physiology and pathology of urologic organs.
\end{abstract}

\section{Keywords}

Urine, Investigations, Urology Practice

\section{Introduction}

Urine examination is one of the important armamentarium in the practice of urology and it is a fundamental test that is usually carried out for urology patients, it can be as simple as dipstick analysis to a complex hormonal assays.

Urine examination is done worldwide as an extension of physical examination which provides a lot of information about the pathology, and both physical and chemical analysis of urine are highly informative [1].

Urine examination should be done before retrograde transurethral procedures to rule out active urinary tract infection. Proper urine is collected when it is indicated that it is paramount to the urologist, however, it can be 
misused when it is done inappropriately or wrongly interpreted.

Urinalysis is one of the most common urine investigations performed in urology practice and it entails the evaluation of physical, chemical and microscopic parameters in the urine. It helps to detect some diseases that may be asymptomatic such as chronic urinary track infections, and it can be performed in hospitals, clinics, accident and emergency department, side rooms or at outpatient department.

With the advent of paper or plastic dipstick microchemistry system that allows for both qualitative and semi quantitative analysis of urine within a short period of time, the colour change seen on the strip is compared with a standard colour chart so as to get actual results. The interpretation of the results depends on the laboratory reference value, the method used for the investigation and the instructions by the manufacturer of the particular test strip.

Urinalysis can be done manually or automated and it involves testing the following parameters: colour, odour, specific gravity, PH, protein, Ketones, glucose, blood cells, Nitrite, bilirubin, urobilinogin, leukocyte esterase, cast, crystals and ova or cyst of parasites.

Methyl red react with hydrogen ions to produce a colour change from red to yellow when the $\mathrm{pH}$ is between 4 \& 6 and bromothymol changes from yellow to blue when the $\mathrm{pH}$ is $6-9$, while the tetrabromophenol blue changes colour from green to blue in the presence of hyperalbuminuria and pseudoperoxidase, activity of haemoglobin catalyzes a reaction between the heme components of both hemoglobin and myoglobin and the chromogentetramethylbenzidine to produce an oxidized chromogen which changes colour from green to blue in hematuria or myoglobin react, glucosidase catalyzes the oxidation of glucose to glucono lactone in the presence of glycosuria, in ketonuria acetoacetic acid in alkaline medium reacts with the sodium nitroprusside to give rise to magenta colour.

In patients with hyperbilirubinemia, urobilinogin reacts with p-dimethylaminobenzaldehyde to change colour from light to dark pink. Nitrite reacts in acid medium with para-arsanilic acid or sulphanilamide to produce a diazonium salt which react with tetrahydrobenzoquinoline to a pink azo dye for rapid detection of infections caused by nitrate reducing bacteria.

Table 1 shows the molecules found in urinalysis.

Table 1. Showing the molecules found in urinalysis.

\begin{tabular}{c} 
Glucose \\
Ketones \\
Bilirubin \\
Urobilinogen \\
Creatinine \\
Catecholamines \\
Dopamine \\
Cortisol \\
Phenylalanine \\
Leukocytes \\
Red Blood Cells \\
Nitrites \\
Proteins \\
Potassium \\
Sodium \\
Chosphate \\
\hline
\end{tabular}


Collection of urine sample in males usually by midstream clean catch, while in females the labia is parted and the vulva swabbed before the sample is taken, or you catheterize the patient and take the sample which is better, and in neonates and infants it is collected by suprapubic tap, adhesive collection bag or by the use of absorbent collection pad [1] [2].

Urine samples should be examined within an hour of collection since allowing the urine sample for longer period will leads to bacterial overgrowth, if the urine cannot be examined immediately then it should be refrigerated at five degrees centigrade [3].

Appropriate use of urine examinations leads to accurate, fast and cost effective diagnosis and management of urologic disease.

A reliable urine test for urothelial cancer may replace or compliment invasive procedures such as urethrocystoscopy, moreover many new urine based tests for urothelial cancer have been developed, some of them such as Bladder Tumor Antigen Stat (BTA), NMP22, FISH and UroVysion are approved by regulatory agencies such as U.S Food and Drug Administration for use while others are in the pipeline [4].

Although dipstick urine test can be fast one has to be wary of false positive and false positive results, especially in the presence of certain drugs such as vitamin C or Captopril hindering the detection of blood in patients with microscopic heamaturia [5].

\section{Types of Urine Investigations}

There are several types of urine investigations used in the practice of urology. And Table 2 shows types of urine investigations used in urology practice.

\subsection{Urinalysis}

This consists of both physical and chemical analysis of the urine.

THE PHYSICAL EXAMINATION OF URINE

This is constituted by the colour, turbidity, specific gravity, osmolality and PH.

COLOUR

Many factors can change the normal amber colour of urine as it is seen.

- Cloudy urine due to the presence of phosphaturia, chyluria or pyuria;

- Red colour urine due to the presence of heamaturia, heamoglobinuria, myoglobinuria, beetroot, drugs such as Rifampicin or phenothiazines;

- Orange colour due to dehydration, phenazophyridine, or sulfasalazine;

- Yellow colour due to phenacetin or riboflavin;

- Green-blue due to the presence of biliverdin, indigo carmine, phenols or methylene blue;

- Brown- black as seen in the presence of alkaptonuria, melanin or sorbitol.

\begin{tabular}{l} 
Table 2. Types of urine investigations used in the practice of \\
urology. \\
\hline Urinalysis \\
Urine Microscopy Culture and Sensitivity (MCS) \\
Urine Macroscopy for Ova or Cyst of Parasites \\
Urine Cytology \\
Urine Tumour Antigens Assays \\
Urine Hormonal Assays \\
Urine Drug Assays \\
Urine Quantitative Measurement \\
Urine Acid Fast Bacilli (AFB) Test \\
Urine Toxicology
\end{tabular}




\section{TURBIDITY}

Presence of turbid urine can be seen in phosphaturia or pyuria usually from infection and rarely from chyluria, other causes of turbid urine include lipiduria, hyperoxaluria and hyperuricosuria.

\section{SPECIPIC GRAVITY}

The normal range of urine specific gravity is 1.001 to 1.035 , it indicates the renal concentrating ability and the hydration status of the patient.

If it is less than 1.008 then it is due to a dilute urine and greater than 1.020 means the urine is concentrated and a fixed urine specific gravity of 1.010 is a sign of renal insufficiency, diabetes and diuretics can reduce the specific gravity while decrease intake and dehydration increase the urine specific gravity [6].

A urine sample with specific gravity greater than 1.035 can be as a result of presence of glucose, dextran or radiopaque dyes.

OSMOLALITY

Measures the amount of materials dissolved in the urine and it varies between 50 and $1200 \mathrm{mosm} / \mathrm{L}$. Urine osmolality is a better indicator of renal function which must be determined using standard laboratory techniques.

$\mathrm{PH}$

The urinary PH ranges from 4.5 to 8 and the average PH is 5.5 - 6.5, hence urine PH is slightly acidic. In patients with metabolic or respiratory alkalosis the urine $\mathrm{PH}$ is alkaline and it can be acidic, in patients with renal tubular acidosis, urine PH can be used in the diagnosis of type of calculus hence as alkaline urine is seen in staghorn calculus while acidic urine can be seen in uric acid and Cystinestones [1] [3].

\section{CHEMICAL EXAMINATION OF URINE}

This involves the evaluation of the biochemical constituents in the urine of the urologic patient and it includes.

\section{URINE DIPSTICKS}

This provides quick and chief methods for detecting abnormal substances in the urine and they usually react to provide calorimetric changes.

Urine dipstick can be used to detect the presence of abnormal substances in the urine such as blood, proteins, glucose, ketones, Bilirubin, white blood cells and nitrites.

A normal plasma proteins and Tamm- Horsfall protein can be found in urine, but not exceeding $150 \mathrm{mg} / 24$ hours or $10 \mathrm{mg} / 100 \mathrm{ml}$ in a single specimen.

The normal glucose level in the urine is usually less than $130 \mathrm{mg} / 24$ hours.

Normal Bilirubin level of the urine is $0.5-1 \mathrm{mg} / \mathrm{dl}$ while the normal Red Blood cells are equal or less than 3 RBCs per high power field.

About 5 hyaline casts are seen normal urine and between 2 - 5 WBCs and upto 20 squamous epithelial cells per high power field.

LIMITATIONS OF URINALYSIS

Some of the limitations of urinalysis include:

1) Presence of some elements may alter the results such a patient on vitamin $C$ which may cause false positive negative nitrite results;

2) Concentrated urine may interfere with interpretation of the colour of the urine;

3) High level of levodopa metabolites may lead to false positive ketone result;

4) Presence of Indican can interfere with bilirubin interpretation;

5) Some Bacterial peroxidase present in urinary tract infection can cause false positive occult blood reaction;

6) Presence of myoglobin may be tested positive for occult blood test;

7) In females the urine sample may be contaminated with vaginal discharge leading to false leukocyte esterase results;

8) High alkaline urine may test false positive for protein;

9) The urine sample can be contaminated with detergents and cause false positive protein results.

\subsection{Urine Microscopy Culture and Sensitivity (MCS)}

This entails detection of bacteria in the urine the type of the bacteria and the sensitivity pattern of the antibiotics tested, this helps in identifying the causative organism of urinary tract infection and the sensitivity pattern to choose the appropriate antibiotic since urinary tract infection is very common [7]. 


\subsection{Urine Microscopy for Ova or Cyst of Parasite}

This involves the microscopic analysis of urine sediments with both low power $(\times 100)$ magnification and high power $(\times 400)$ magnification lenses.

LOW POWER MAGNIFICATION: this is used to identify the presence of crystals and ova of parasites such as the schistosoma ova which have a terminal spine. Trichomonas vaginalis can also be detected by low power magnification.

HIGH POWER MAGNIFICATION: this is used to distinguish between circular and dysmorphic erythrocytes as seen in nephrologic and urologic heamaturia, it can also helps in types of crystals and differences between bacteria and yeast.

\subsection{Urine Cytology}

Urine cytology involves the use of microscope to detect presence of exfoliated malignant cells from the bladder especially transitional cell carcinoma.

The urine sample is obtained early morning or at cystoscopy using barbotage which increases the yield of detecting the urothelialcancer [1]-[3].

\subsection{Urine Tumour Antigens Assay}

The urine tumour antigens such as Bladder tumour antigen (BTA), Nuclear matrix protein 22 (NMP22) and Quanticyst are all bladder tumour antigens which can be assayed in the urine.

The urine prostate antigen 3 (PCA3) is a promising biomarker in the diagnosis of cancer of the prostate with a specificity approaching $100 \%$, this antigen is expressed in prostate cancer tissue 66 - 100 times more than in normal prostate tissue and 140 times more than in Benign Prostatic Hyperplasia (BPH). Vincenzo et al. assayed PCA 3 of 108 patients and got the following results sensitivity of $67 \%$, specificity of $83 \%$, positive predictive value of $53 \%$ and negative predictive value of $90 \%$ [8].

\subsection{Urine Hormonal Assay}

Certain hormones can be assayed in the urine such as vanillymandelic acid, metanephrine, epinephrine and norepinephrine in patients with pheochromocytoma, high level of aldosterone in the urine is seen in aldosterone secreting tumour and adrenocortical tumours may be detected by the presence of high level of urinary 17-ketosteroids [9] [10].

\subsection{Urine Toxicology}

Some drugs can be assayed in the urine of urologic patients such as immunosuppressive drugs in renal transplant patients where the level of urinary tacrolimus is assayed which will indicate whether the patient is developing tacrolimus toxicity and hence the need to reduce the dose of the drug or if the level is lower than 4 indicating the need to increase the dose to prevent rejection. Other drugs that can be assayed in urologic patient include substance of abuse seen in some patients with priapism [11].

\subsection{Urine Quantitative Measurement}

A 24 hours urine collection is done to determine the quantity of the urine output of the urologic patient to ascertain the renal function.

\subsection{Urinary Acid Fast Bacilli Test}

The urinary Acid Fast Bacilli (AFB) Test is done in patients suspected to have genitourinary tuberculosis and usually six bottles of sterile containers are used to collect the urine samples over six days.

\section{Uses of Urine Examinations in Urologic Practice}

The uses of urine examinations in urology practice can be broadly classify into three diagnostic, monitoring and prognostication uses. 
Diagnostic Uses includes:
a) Renal Function Test;
b) Evaluation of heamaturia;
c) Evaluation of urolithiasis;
d) Evaluation of urinary tract infections;
e) Evaluation of urologic cancers;
f) Evaluation of urinary fistulae;
g) Evaluation of infertility;
h) Evaluation of inborn errors of metabolism.
Monitoring Uses includes:

a) Monitoring of urologic malignancies;

b) Monitoring of post obstruction diuresis;

c) Monitoring of renal transplant patients;

d) Monitoring of patients with urinary tract infections;

Prognostication Uses includes:

a) Prognostication of patients with acute tubular necrosis;

b) Prognostication of renal function in patients with oliguria;

c) Prognostication of patients with obstructive nephropathy.

Table 3 shows the Diagnostic uses of urine investigations in urology.

\subsection{Renal Function Test}

This entails measuring the urine specific gravity which decreases progressively in patients with renal impairment due to loss of concentrating ability of the kidney.

The measurement of urine osmolality is another measurement of renal function which is a better parameter of measuring the renal function.

Twenty four hours urine collection and measurement of urinary creatinine is another measure to the function of the kidney and in patients with chronic renal failure there will be persistence of more than 30 grams of albumin per gram creatinine in the urine.

The glomerular filtration rate (GFR) uses the urine creatinine concentration, the urine flow rate and the plasma concentration can be used to calculate the GFR using the formula [3].

$$
\text { GFR }=\frac{\text { Urine Concentration of Creatine } \times \text { Urine Flow }}{\text { Plasma Concentration of Creatinine }}
$$

\subsection{Evaluation of Heamaturia}

Presence of 3 Red Blood Cells (RBS) per high power field indicates microscopic heamaturia which is seen in some urologic pathology.

The red colour of urine may indicates heamaturia especially if microscopically erythrocytes are demonstrated. In urologic diseases the presence of normal RBCs are seen in the urine which differentiates it from Nephrological causes of heamaturia where dysmorphic RBCs are demonstrated [12].

Table 3. Diagnostic uses of urine investigations in urology.

Renal Function Test
Evaluation of Heamaturia
Evaluation of Stone Diseases
Evaluation of Urinary Tract Infections
Evaluation of Urologic Cancers
Evaluation of Urinary Fistulae
Evaluation of Infertility
Evaluation of Inborn Errors of Metabolism


Urine dipstick test can also differentiate the reddish discolouration of urine due to heamaturia from myoglobinuria or metheamalgobinuria and the use of an automated system iQ200 has been shown to be accurate for detecting, enumerating and sizing RBCs in urine [13] [14].

\subsection{Evaluation of Urolithiasis}

Macroscopy of urine is very important evaluating patients with urolithiasis since various forms of crystals are characteristics of different types of stones, example include uric acid crystals seen in uric acid stone, triple phosphate crystals in struvite stones, Indinavir crystals which is characteristically flat and rectangular plates often in fan or star burst pattern seen in patients on Indinavir, and the PH of urine is important in determining the types of stone the patient has such as uric acid stones are formed in acidic urine while struvite stones are formed in alkaline urine [15]-[17].

\subsection{Evaluation of Urinary Tract Infections}

The urine examination is paramount in evaluating patients with urinary tract infections, the use of flow cystometry based urine analysis can detect bacteria with nearly $80 \%$ accuracy.

The urine culture can be used to estimate the number of bacteria in the urine to identify the exact causative organism and the sensitivity pattern to appropriate drug use.

The presence of 5 leucocytes per high power field is generally considered as pyuria due to infection although renal tuberculosis can cause sterile acid pyuria with negative results on routine bacterial cultures however a microscopic examination using fluorescent stain can demonstrate acid fast bacilli (AFB) in genitourinary tuberculosis [18]-[20].

\subsection{Evaluation of Urologic Cancers}

The urine cytology is used in the diagnosis of high grade urothelial bladder cancers assay of BTA is promising as a non invasive test for bladder cancer.

PCA 3 is gaining ground in diagnosis of prostate cancer so also urine vinyl mendelic acid is used in the diagnosis of Pheochromocytoma.

Elevated levels of urinary 17-ketosteriods are seen in patients with adrenocortical tumours and high level of epinephrine in neuroblastoma.

\subsection{Evaluation of Urinary Fistulae}

Discolouration of urine after ingestion of a poorly absorbed dye such as phenol red will confirm the diagnosis of fistula of the urinary tract and bowel.

The 3 swab test in a female patient with suspected urinary fistula where a methylene blue is instilled into the bladder and indigo carmine given intravenous which will help distinguish between the ureterovesical and vesicovaginal fistulas.

\subsection{Evaluation of Infertility}

In patients with retrograde ejaculation as the cause of infertility post coital urine microscopy may demonstrate the presence of sperm cells in the urine.

\subsection{Evaluation of Inborn Errors of Metabolism}

Patients with some inborn error of metabolism leading to formation of stones such as presence of cystine crystals in cases of cystinuria or calcium oxalate crystals in case of oxaluria.

Table 4 shows the uses of urine investigations in monitoring.

\subsection{Monitoring of Urologic Malignancies}

Certain urologic malignancies can be monitored by assaying the urine level of their tumour markers such as patients with Pheochromocytoma the urine assay of vinyl mendelic acid is used to monitor the patient post operatively. 
Table 4. Uses of Urine investigations in monitoring.

\author{
Monitoring of Urologic Malignancies \\ Monitoring of Post Obstruction Diuresis \\ Monitoring Renal Transplant Patients \\ Monitoring Patients with Schistosomiasis \\ Monitoring Patients with Urinary Tract Infections
}

Nuclear Matrix Protein 22 is used to monitor patients with urothelial bladder tumour post cystectomy.

The level of urinary 17-ketosteroids is used to monitor patients with adrenocortical tumours while the level of urinary Prostate Cancer Antigen is used to monitor patient with Prostate cancer.

\title{
3.10. Monitoring of Patients with Post Obstruction Diuresis
}

Urine investigations are very important in monitoring patients with post obstruction diuresis, the quantity of urine the patient is making is used to monitor the progression or otherwise of post obstruction diuresis where the patient can make up to eleven litres of urine within 24 hours.

The specific gravity of the urine is also used to monitor the return of renal concentrating ability in such patients, in normal individuals the specific gravity of urine is $1.003-1.030$ but in patients with post obstruction diuresis it can be as low as 1.010 .

\subsection{Monitoring Renal Transplant Patients}

In post transplant patient the actual amount of urine made by the kidney per hour is very important as such is used to monitor the function of the kidney presence or absence of delay graft function in which the patient may have dialysis before the kidney picks up.

The use of urine creatinine level will determine in conjunction with the serum creatinine level where the patient is having slow graft function.

\subsection{Monitoring the Patients with Schistosomiasis}

In patients with vesical Schistosomiasis the use of urine microscopy to look for the ova of schistosomaheamatobium is used to monitor the patient on treatment which will determine the need or otherwise of repeating the treatment depending on the presence or absence of the ova of Schistosomiasis in the urine of the patients.

\subsection{Monitoring Patients with Urinary Tract Infections}

The use of microscopy culture and sensitivity test of urine is very important in monitoring urologic patients with urinary tract infections, while the patient is on treatment the urine examination is repeated to ascertain the eradication of the infecting microorganism in the urinary system.

\subsection{Uses of Urine Examination for Prognostication}

The use of specific gravity of urine for prognostication of patients with acute tubular necrosis in which if the specific gravity is consistently 1.010 it means the prognosis is not good as the patient must have suffered extensive renal tubular damage.

The use of urine volume in determining the renal function and the prognosis of the likely outcome after challenging the kidney for example in a patient with persistent oliguria which is the urine volume of $400 \mathrm{mls}$ per 24 hours indicating the level of renal failure.

Urine protein level is also used to prognosticate about the patient with obstructive nephropathy.

\section{Conclusion}

The uses of urine investigations in urologic practice cannot be over emphasized as it has a lot of revealing information as to the physiology and pathology of urologic patients, and when urine investigations are misused, it 
can lead to inappropriate treatment of the patient. Therefore there is need to standardise urine investigations and correlated clinically to achieve better results.

\section{The Author}

Dr. Muhammad Ujudud Musa MBBS, FWACS, is a consultant urologist in the Department of Surgery, Federal Medical Centre Katsina, Katsina State Nigeria, a clinical researcher and a trainer of Resident Doctors.

\section{Complete of Interest}

The author declared no complete of interest and no grant received for this article.

\section{References}

[1] Gerber, G.S. and Brendler, C.B. (2012) Evaluation of the Urologic Patient: History, Physical Examination and Urinalysis. In: Wein, A.J., Kavoussi, L.R., Novick, A.C., Partin, A.W. and Peters, C.A., Eds., Campbell-Walsh Urology, 10th Edition, Chapter 3, Elsevier Saunders, Philadelphia, 87-88.

[2] O’Toole, S.J. (2008) Urinary Tract Infections. In: Thomas, D.F.M., Duffy, P.G. and Rickwood, A.M.K., Eds., Essentials of Paediatric Urology, 2nd Edition, Chapter 4, Informa Healthcare, London, 43.

[3] Kreder Jr., K.J. and Williams,R.D. (2008) Urologic Laboratory Examination. In: Tanagho, E.A. and McAninch, J.W., Eds., Smith's General Urology, 17th edition, Chapter 5, McGraw Hill Lange, New York, 46.

[4] van Rhijin, B.W.G., van der Poel, H.G. and van der Kwast, T.H. (2009) Cytology and Urinary Markers for the Diagnosis of Bladder Cancer. European Urology Supplements, 8, 533-541.

[5] Hohenfellner, M. and Santucci, R. (2007) The Clinical Approach to the Acutely Ill Patient. In: Hohenfellner, M. and Santucci, R.A., Eds., Emergencies in Urology, Chapter 2, Springer-Verlag Berlin, Heidelberg, 4. http://dx.doi.org/10.1007/978-3-540-48605-3

[6] Simerville, J.A., Maxted, W.C. and Palurra, J.J. (2005) Urinalysis: A Comprehensive Review. American Family Physician, 71, 1153-1162.

[7] Wagenlehner, F.M., Pilatz, A., Naber, K. and Weidner, W. (2014) Urinary Tract Infections. Aktuelle Urologie, 45, 135-145.

[8] Ficcara, V., Novara, G. and Zattoni, F. (2010) The Role of Prostate Cancer Antigen e (PCA3) Test for the Diagnosis of Prostatic Cancer in the Era of Opportunistic Prostate Specific Antigen Screening. European Urology, 58, 482-484. http://dx.doi.org/10.1016/j.eururo.2010.07.025

[9] Hoff, W.V., Bicknell, E.J., Horrocks, P.M., Fleetwood, J.A., Inthuprapa, M. and Hall, R. (1977) Urinary Pregnanetriol Excretion in Hirsutism. Clinica Chimica Acta, 80, 373-379.

[10] Escamilla, R.F. (1949) Diagnostic Significance of Urinary Hormonal Assays: Report of Experience with Measurements of 17-Ketostreoids and Follicle Stimulating Hormone in the Urine. Annals of Internal Medicine, 30, 249-290. http://dx.doi.org/10.7326/0003-4819-30-2-249

[11] Standridge, J.B., Adams, S.M. and Zotos, A.P. (2010) Urine Drug Screening: A Valuable Office Procedure. American Family Physician, 81, 635-640.

[12] Bagnall, P. (2014) Haematuria: Classification, Causes and Investigations. British Journal of Nursing, 23, $1074-1078$. http://dx.doi.org/10.12968/bjon.2014.23.20.1074

[13] Turkeri, L., Mangir, N., Gunlusoy, B., Yildirim, A., Baltaci, S., Kaplan, M., Bozlu, M. and Mungan, A. (2014) Identification of Patients with Microscopic Hematuria Who Are at Greater Risk for the Presence of Bladder Tumors Using a Dedicated Questionnaire and Point of Care Urine Test-A Study by the Members of Association of Urooncology. Turkey. Asian Pacific Journal of Cancer Prevention, 15, 6283-6286. http://dx.doi.org/10.7314/APJCP.2014.15.15.6283

[14] Dal Moro, F., Valotto, C., Guttilla, A. and Zattoni, F. (2013) Urinary Markers in the Everyday Diagnosis of Bladder Cancer. Urologia, 80, 265-275. http://dx.doi.org/10.5301/urologia.5000041

[15] Pak, C.P.C., Peterson, R. and Poindexter, J.R. (2001) Adequacy of a Single Stone Risk Analysis in the Medical Evaluation of Urolithiasis. The Journal of Urology, 165, 378-381. http://dx.doi.org/10.1097/00005392-200102000-00006

[16] Portis, A.J. and Sundaram, C.P. (2001) Diagnosis and Initial Management of Kidney Stones. American Family Physician, 63, 1329-1339

[17] Park, S. and Pearle, M.S. (2005) Urolithiasis: Update on Metabolic Evaluation of Stone Formers. The Scientific World Journal, 5, 902-914. http://dx.doi.org/10.1100/tsw.2005.118 
[18] Jarvis, T.R., Chan, L. and Gottlieb, T. (2014) Assessment and Management of Lower Urinary Tract Infection in Adults. Australian Prescriber, 37, 7-9.

[19] Kapoor, R., Ansari, M.S., Anil Mandhani, A. and Gulia, A. (2008) Clinical Presentation and Diagnostic Approach in Cases of Genitourinary Tuberculosis. Indian Journal of Urology, 24, 401-405. http://dx.doi.org/10.4103/0970-1591.42626

[20] Çek, M., Lenk, S., Naber, K.G., Bishop, M.C., Johansen, T.E.B., Botto, H., Grabe, M., Lobel, B., Redorta, J.P. and Tenke, P. (2005) EAU Guidelines for the Management of Genitourinary Tuberculosis. European Urology, 48, 353362. 